www.jmscr.igmpublication.org Impact Factor 5.244

Index Copernicus Value: 83.27 ISSN (e)-2347-176x ISSN (p) 2455-0450 crossref DOI: _https://dx.doi.org/10.18535/jmscr/v4i11.85

Journal Of Medical Science And Clinical Research

\title{
Potentially Inappropriate Medication Use among Elderly Inpatients at a Teaching Hospital in South India
}

\author{
Authors \\ Dr Steve Paul Manjaly ${ }^{1}$, Dr Geetha Francis ${ }^{2}$, Dr Betsy Mathew ${ }^{3}$ \\ ${ }^{1}$ MBBS. DNB (Medicine), DGM (Geriatric Medicine), Assistant Professor, Department of Medicine, Jubilee \\ Mission Medical College and Research Institute, Thrissur, Kerala \\ ${ }^{2} \mathrm{MD}$ (Medicine), Associate Professor, Department of Medicine, St John's Medical College, Bangalore \\ Email: francis.geetha@gmail.com \\ ${ }^{3}$ MBBS MD (Medicine), DGM (Geriatric Medicine), Professor and Head, Division of Geriatric Medicine, \\ Department of Medicine, St John's Medical College, Bangalore \\ Email: betsy123m@hotmail.com \\ Corresponding Author \\ Dr Steve Paul Manjaly \\ MBBS. DNB (Medicine), DGM (Geriatric Medicine), Assistant Professor, Department of Medicine, Jubilee \\ Mission Medical College and Research Institute, Thrissur, Kerala
}

\begin{abstract}
BACKGROUND: Use of inappropriate medications among elderly is common. An association between potentially inappropriate medication (PIM) use in elderly and adverse outcomes such as impaired muscle strength, functional status, increased healthcare expenditure, and increased risk of hospitalization and death has been demonstrated. Yet there are few studies addressing this issue.

OBJECTIVE: This study focuses on the prevalence of the use of potentially inappropriate medications in hospitalized elderly.

DESIGN: A retrospective observational study was done between June and December 2012 involving 150 patients. Patient data including period of hospital stay, medical illnesses and medication list at discharge were obtained from the in-patient records. The chi-squared test for categorical variables was used to compare the characteristics of participants receiving and not receiving inappropriate medications.

SETTING: The study collected data from 150 patients admitted to a teaching hospital at Bangalore, Karnataka.

PATIENTS: The sample was composed of 150 patients aged $>60$ years admitted with multiple co morbidities. MEASUREMENTS: The percentage of patients prescribed PIMs as defined using a modified Beers list was measured. Multivariable-adjusted odds ratios for PIM use were computed.

RESULTS: Of the 150 patients, $17.3 \%$ received at least 1 PIM, and $7 \%$ received 3 or more. Most common category of PIM use were Prazosin, followed by benzodiazepines

PIM use was directly associated with no: of comorbidities and Polypharmacy

CONCLUSION: There is a widespread use of potentially inappropriate medications among elderly patients in the population studied. Physicians should be more aware of the Beer's criteria when treating the elderly.

KEYWORDS: Potentially inappropriate medications (PIM), Beers criteria (BC), Polypharmacy, geriatric, elderly.
\end{abstract}




\section{INTRODUCTION}

India, now home to 1.2 billion people, is projected to overtake China in about a decade to become the world's most populous country. Between 2010 and 2050, the number of those aged above 60 years is also is expected to increase from 7 percent to 14 percent of the total population. Presently we have about 100 million elderly in India. Elderly suffer from multiple illnesses both acute and chronic, requiring multiple hospital visits and medications from multiple specialists.

Medications can be considered inappropriate when their risk outweighs their benefit. The pharmacokinetics and pharmacodynamics of drugs is different in elderly and knowledge of geriatric pharmacology is of utmost importance in prescribing for the elderly.

The Beers criteria, named after Mark Beers, MD, lists medications that should generally be avoided in older adults. First evolved in 1991 by 13 pharmaco therapeutic and geriatric specialists as a tool for safer medication prescribing in frail older adults residing in nursing homes, it was revised in 1997 and again in 2003 to apply to all persons aged $\geq 65$ years, and was most recently updated in 2012 . The final updated Beers criteria encompasses fifty-three medications or medication classes, which are divided into three categories: potentially inappropriate medications (PIM) and classes to avoid in older adults, those to avoid in older adults with certain diseases and syndromes that these drugs can exacerbate, and finally medications to be used with caution in older adults. ${ }^{1},{ }^{\mathrm{I}}$

Polypharmacy (defined as the concurrent use of multiple medications) is common in the elderly and its prevalence ranges from $33 \%$ to $58 \% .^{1,2}$ Unfortunately, polypharmacy may cause problems such as drugdrug interactions, non-adherence to therapy and adverse drug effects.

\section{Objective of Study:}

An association between potentially inappropriate medication (PIM) use in elderly and adverse outcomes such as impaired muscle strength and functional status, increased healthcare expenditure, and increased risk of hospitalization and death has been demonstrated. ${ }^{345}$ Use of inappropriate medications among elderly is common both in ambulatory and hospitalized settings. ${ }^{6}$ Yet there are few studies addressing this issue among the elderly in India.

Our objectives were to study the prevalence and predictors of the use of potentially inappropriate medications in hospitalized elderly and the associations between PIM use and comorbidities, polypharmacy, and duration of hospitalization.

\section{MATERIALS AND METHODS}

This is a retrospective study conducted in a 1200 bedded tertiary care teaching hospital in Karnataka, South India.

150 patients discharged from medical wards between June and December 2012 were included. Patient data including period of hospital stay, medical illnesses and medication list at discharge were obtained from the in-patient records.

Wherever the medication list at discharge was unavailable, medications ordered on the last day of admission were analyzed. The medication appropriateness was decided according to Beers criteria. The number of 
discharge medications included not only oral and parenteral medications but also inhalers, and topical medicines. Combination medications were counted separately; insulin and multivitamins were counted as a single medication.

The patients were grouped according to their age into young old (65-74 yrs), old old (75-85yrs) and oldest old (>85 yrs). The presence of chronic medical illnesses like diabetes, hypertension, chronic obstructive pulmonary disease, cerebrovascular disease and ischemic heart disease were identified and the use of inappropriate medications was assessed in each group.

Whenever a potentially inappropriate medicine was identified, it was classified according to the Beers criteria into the three categories mentioned above. The drugs were not counted as inappropriate if they were used in a palliative setting or if there was no other alternative to its use.

Polypharmacy was considered as the administration of $\geq 5$ drugs per day.

Data was assessed using the statistical package for social sciences, version 16. The chi-squared test for categorical variables was used to compare the characteristics of participants receiving and not receiving inappropriate medications. A probability value of less than 0.05 was considered statistically significant.

\section{RESULTS}

\section{Patient characteristics (Table 1)}

A total of 150 patient records were analyzed. Out of the total 150 patients, $120(80 \%)$ were aged 60-74 years (young old), $22(14.7 \%)$ were in the age group of 75-84 years (old old), and 8 (5.3\%) were aged above 85 years (oldest old). The mean age of the patients in the study group was 67.8 years. Of the 150 patients, 56 $(37.3 \%)$ were females and $94(62.6 \%)$ were males.

The average number of chronic co-morbid medical illnesses per patient was $4.28( \pm 2.235)$. The commonest co-morbid illnesses were hypertension $(n=80)(53 \%)$ and diabetes $(n=78)(52 \%)$.

The average duration of hospital stay was 8.06 days (mean duration $=8$ days)

\section{Polypharmacy (Table 2)}

$110(73.3 \%)$ ) patients were subject to polypharmacy.. The mean number of medications consumed per day by the patients in the study group was $6.7 \pm 3.1$. 40 patients consumed $<5$ medications, 84 consumed between 5 to 10 medications, and 26 regularly consumed $\geq 10$ medications per day.

The average number of medications taken by elderly men $(n=94)$ was $6.79( \pm 3.2)$ per day and elderly women $(n=56)$ were taking $6.62( \pm 2.9)$ medications per day. The patients of the oldest old category $(\geq 85$ years $n=8)$ had an average of 7.38 medications/day. The young old $(n=120)$ had a mean of 6.72 medications per day and the old old $(n=22)$ had a mean of 6.5 medications per day.

The prevalence of polypharmacy was higher in those patients who had multiple co morbid illnesses. 110 patients were subject to polypharmacy. A patient who was subject to polypharmacy had an average of 4.9 comorbid illnesses, whereas the others had an average of 2.5 comorbid illnesses.

Potentially inappropriate medications (Table 3)

Of the total number of 150 patients in the study group, one of six (26) were taking a potentially inappropriate medication according to the Beers' list. $(\mathrm{p}=0.830)$

Of the 26 patients taking a PIM, men constituted the majority, 17 (65.3\%) compared to women, 9 (34.7\%). Eighteen of them belonged to the young old $(n=120), 3$ of the old old $(n=22)$ and 5 of them belonged to the oldest old category $(n=8)$.

Nineteen (73\%) were prescribed a single inappropriate medication, one of five (5) patients were prescribed 2 potentially inappropriate medicines and one of thirteen (2) were taking 3 drugs that were potentially inappropriate. 
Among the potentially inappropriate medications, the most common ones belonged to those that acted on the cardiovascular system, (13) (50\%), of which antihypertensives (prazocin) constituted the majority (6)(46\%) followed by anti arrhythmic ( amiodarone)(5) (38.4\%) and vasodilators (hydralazine and nitrates)(2)(15.3\%) . The second commonest inappropriately prescribed drugs were those that acted on the central nervous system (30\%), chiefly benzodiazepines (6) ( alprazolam) followed by anticholinergics. (amitryptiline).

The maximum duration of hospital stay by any patient was 55 days, $(0.7 \%)$ with most patients staying on an average duration of 8.1 (range 1- 55). PIM users stayed on a higher average of 8.7 days when compared to the non- PIM users. $($ Mean=7.93) $(\mathrm{p}=0.602)$

The average number of medical illnesses seen in the group inappropriately prescribed was 5.7 (4.7, 6.7), whereas the group without any inappropriate medication was $3.97(3.6,4.3)(\mathrm{p}<0.001)$.

Of the 26 PIM users, majority (22) (84.6\%) were hypertensives (Odds Ratio 6.3; C.I 1.9-22.8), 10 (38.4\%) had Ischemic heart disease,( Odds ratio 3.9; C.I 1.4-11.1), 18 (69\%) had diabetes, and the proportion of patients with chronic obstructive pulmonary disease and cerebrovascular disease were $9(34.6 \%)$ and 2 (7.6\%) respectively. Of these, Hypertension, (84.6\%) $(\mathrm{p}<0.001)$ ischemic Heart disease $(38.4 \%)(\mathrm{p}=0.009)$ were significantly associated with the use of inappropriate medications.

The prevalence of PIM use varied according to the co morbid illnesses. PIM use was highest in those with ischemic heart disease $(10$ of 27$)(37 \%)(\mathrm{p}=0.009)$, hypertension $(22$ of 80$)(27.5 \%)(\mathrm{p}=0.000)$ followed by diabetes $(18$ of 78$)(23 \%)(\mathrm{p}=0.053)$ and chronic lung disease $(2$ of 13$)(15 \%)(\mathrm{p}=0.88)$

$\mathrm{PIM}$ and polypharmacy

Of the 110 patients who were subject to polypharmacy, $21(19.09 \%)$ were using an inappropriate medication. Of the 40 patients who were not on polypharmacy, five had PIM use. $(p=0.34)$

The maximum number of medications a patient was prescribed was 16. (Range 1-16, Mean $6.73 \pm 3.09$ ) 25 were prescribed $\geq 10$ medicines, and 85 were prescribed $\geq 5$ medicines. Of the 26 PIM users, $21(80.7 \%)$ were subject to polypharmacy with an average of 8 medicines (mean $8.12 \pm 3.36$ ) as compared to the non PIM users (Mean $6.44 \pm 2.92$ ).

\section{DISCUSSION}

The study demonstrates that of the total number of patients discharged from medical wards, $17.3 \%$ were taking at least one inappropriate medication. $(\mathrm{p}=0.830)$. The prevalence of PIM use was significantly associated with increasing age $(\mathrm{p}<0.001)$

The prevalence of PIM use was lower than in international studies conducted on the use of inappropriate medications conducted among hospitalized elderly patients in Ireland $(32 \%)^{8}$, France $(66 \%),{ }^{9}$ Switzerland $(22.1 \%)^{10}$, and Taiwan $(23.7 \%)^{11}$. Among studies conducted in India, our results were lower compared to ones conducted in other teaching hospitals in Karnataka- Bangalore ${ }^{12}$, Mysore ${ }^{1}$ but comparable to studies conducted in Punjab (18\%) ${ }^{2}$. The difference could be probably due to the differences in patient and disease characteristics, prescribing patterns, study settings and availability of medications listed in BC.

As in other studies, the prescription of inappropriate medication had a significant association with increasing co morbidity. ${ }^{3}$. The average number of medical illnesses seen in the group inappropriately prescribed was 5.77, whereas the group without any inappropriate medication was $3.97 \pm 2.05)$ Hypertension, $(84.6 \%)(\mathrm{p}$ 
$<0.001)$ ischemic Heart disease $(38.4 \%)(\mathrm{p}<0.001)$ were significantly associated with the use of inappropriate medications

Among the chronic illnesses, Essential hypertension and Ischemic heart disease increased the odds of use of PIM, similar to other studies in India 205

The most commonly prescribed inappropriate medication in our study were antihypertensives, chiefly prazocin (46\%), antiarrhythmics, amiodarone (38\%) followed by drugs acting on the CNS, benzodiazepines $(19 \%)$, and anticholinergics (15\%). This was different from other studies ${ }^{19-21,23}$ where drugs acting on the respiratory system comprised the majority of the inappropriate medications. The difference was probably due to the increased prevalence of hypertensives (53\%) in the population.

As in other studies, prescription of inappropriate medications had a statistically significant association with increasing age (>84 years), ${ }^{21}$ with 5 of 8 patients receiving inappropriately prescribed medications. (p $<0.001)$.

Contrary to other studies, ${ }^{21,22,23}$ we did not find any statistically significant association with inappropriate medication use and duration of hospital stay.

Studies have shown that use of inappropriate medications is significantly associated with increased duration of hospital stay. ${ }^{21,22,23}$ We did not find such an association, This could be attributed to improved drug monitoring or other factors that could influence duration of hospital stay e.g social factors, costs of hospitalization etc.

\section{Polypharmacy}

Our study found a higher prevalence of polypharmacy; compared to previous studies ${ }^{1}$ in northern India 110 $(73.3 \%)(\mathrm{p}<0.001)$. The ninety -four elderly men in the study population consumed a higher average number of medications compared to that of the elderly women (mean 6.85 vs 6.53 , respectively). ( $p>0.001$ )

The presence of diabetes, $(n=78$; mean 7.26), hypertension $(n=80 ;$ mean 7.9), ischemic heart disease $(n=27$; mean 8.07) and chronic pulmonary illness $(n=33$; mean 7.97) had a statistically significant association with polypharmacy use when compared to the patients with other co morbid illnesses. Of these, hypertension appeared to be the most important predictor of polypharmacy $(\mathrm{p}<0.001)$

A higher prevalence of potentially inappropriate medication use was seen among those with increasing number of medications. 26 patients who were taking inappropriate medicines were prescribed a higher average number of medications (mean 8.12 \pm 3.36 ) when compared to among the 124 non PIM users. (Mean $6.44 \pm 2.92)(\mathrm{p}<0.02)$

Of the 26 patients who were inappropriately prescribed, $21(80.7 \%)$ were subject to polypharmacy, although they formed only $19 \%$ of the study population ( $\mathrm{p}$ 0.346)

Inappropriate prescription was directly linked to polypharmacy. The prescription of inappropriate medications increased as the number of concurrent medications increased. This was similar to studies conducted in other hospital in Karnataka ${ }^{19,20}$. When polypharmacy was defined as the use of 5 or more drugs, this association differed markedly. Only 21 of the total 110 were prescribed more than 5 medications, which was statistically insignificant, suggesting that a quantitative definition for polypharmacy was questionable. $^{6}$

Polypharmacy was however rampant in the study group, as is known from previous studies ${ }^{78}$, and this was directly linked to morbidity in our study group.

This study is important because it shows that despite the existence of Beers criteria since 1987 and its regular updation, last in 2012, it still has not found its way into mainstream practice. Hence, the elderly continue to be prescribed those medicines that are known to have adverse effects in them. Every adverse event could be misdiagnosed and another drug added to alleviate the new symptom and this leads to the 
prescribing cascade, ${ }^{9}$ subsequently adding to polypharmacy. Patients are at an increased risk of receiving an inappropriate medication and having an adverse drug reaction (ADR), which may impact a patient's adherence to his or her medication regimen. Polypharmacy has also been reported to increase the risk of geriatric syndromes and morbidity/ mortality. ${ }^{27,10}$

The prevalence of the use of inappropriate medications is a concern, because studies have shown to have negative consequences such as increased risk of falls ${ }^{11}$, impaired activities of daily living ${ }^{12}$, non adherence to medication ${ }^{13}$ and increased medical costs. ${ }^{14}$

The increased prescription of inappropriate medications in patients with hypertension and diabetes points to the increased prevalence of these illnesses in the Indian elderly population. Although our study did not prove any statistical association, the use of inappropriate medications increases with the increased duration of hospital stay ${ }^{23}$, and was known to have significant association with adverse effects such as falls ${ }^{32}, 15$, and cognitive dysfunction ${ }^{16}$.

Our study also had certain limitations. This was a retrospective study, based on a small sample size of 150 patients, with information on drugs prescribed obtained from case records. The drug prescription was obtained from the discharge summaries, and where the same was not available, from the last day of inpatient admission. This may not have been a true representation of inappropriate medication use, since the medication list at discharge would have largely been modified to suit the chronic illness, rather than during the acute period.

The prescription of inappropriate medicine among the elderly was based on the updated Beers' criteria 2012. Beers' criteria do not address several other important aspects of inappropriate prescribing in older people, e.g. duplicate drug class prescriptions, harmful drug-drug interactions, inappropriate duration and frequency of therapy, and perhaps most importantly, drugs that are often omitted from older people's prescriptions. Beers' criteria are not organized in such a way as to make them quick and easy for the busy prescribing physician or dispensing pharmacist to use. Further, errors in prescription writing, errors of commission and omission were not looked into. Other tools for drug prescription in the elderly such as START ${ }^{17}, \mathrm{STOPP}^{18}$ and IPET ${ }^{19}$ (Improved Prescription in the Elderly Tool) would provide more insight on the use of inappropriate prescriptions in the elderly.

Our data was obtained from patients admitted and discharged from medical wards. A review of the discharge medications of patients admitted from surgical specialties would probably have shown different results. Patients with chronic illnesses like osteoarthritis, osteoporosis were not represented adequately, not only because such patients visited the orthopedic department more frequently, but also because they were probably undiagnosed in the medical wards.

Adverse drug events can be a cause and effect of inappropriate medication use. There was no data obtainable from the discharge records, and hence drug related events to inappropriate medications could not be studied.

\section{CONCLUSION}

Pharmacotherapy in the elderly requires a balance between inappropriate medication use and undertreatment. Interventions aimed at rational medication use in elderly Indians should focus on the predictors of use of inappropriate drugs. The need of physicians trained in Geriatric medicine is rising due to the changing patient characteristics in the Indian population scenario. Geriatricians are more likely to be aware of geriatric pharmacotherapy and its effects ${ }^{20}$. There is a great scope for conducting intense research to determine inappropriate medication use and its health-related adverse consequences in the increasing Indian elderly population. 


\section{REFERENCES}

1. Campanelli CM. American Geriatrics Society Updated Beers Criteria for Potentially Inappropriate Medication Use in Older Adults: The American Geriatrics Society 2012 Beers Criteria Update Expert Panel. J Am Geriatr Soc. 2012; 60(4):616-631.

2. John et al, A study on polypharmacy in senior Indian population, ijpcbs 2013, 3(1), 168-171.

3. Lau DT, Kasper JD, Potter DE, Lyles A, Bennett RG. Hospitalization and death associated with potentially inappropriate medication prescriptions among elderly nursing home residents. Arch Intern Med. 2005;165:68-74.

4. Landi F, Russo A, Liperoti R, et al. Impact of inappropriate drug use on physical performance among a frail elderly population living in the community. Eur J Clin Pharmacol. 2007;63:791-799

5. Fu AZ, Liu GG, Christensen DB. Inappropriate medication use and health outcomes in the elderly. J Am Geriatr Soc. 2004;52:1934-1939.

6. Aparasu RR, Fliginger SE. Inappropriate medication prescribing for the elderly by office-based physicians. Ann Pharmacother 1997;31:823-9.

7. Goulding, Inappropriate Medication Prescribing for Elderly Ambulatory Care Patients, Arch Intern Med. 2004;164(3):305-312

8. Barry PJ, O'Keefe N, O'Connor KA et al. Inappropriate prescribing in the elderly: a comparison of the Beers criteria and the improved prescribing in the elderly tool (IPET) in acutely ill elderly hospitalised patients. J Clin Pharm Ther 2006; 31: 617-26.

9. Laroche ML, Charmes JP, Nouaille Y, Fourrier A, Merle L. Impact of hospitalisation in an acute medical geriatric unit on potentially inappropriate medication use. Drugs Aging 2006;23:49-59.

10. Egger SS, Bachmann A, Hubmann N, Schlienger RG, Krohenbóhl S. Prevalence of potentially inappropriate medication use in elderly patients: Comparison between general medical and geriatric wards. Drugs Aging 2006;23:823-37.

11. Lin HY, Liao CC, Cheng SH, Wang PC, Hsueh YS. Association of potentially inappropriate medication use with adverse outcomes in ambulatory elderly patients with chronic diseases: Experience in a Taiwanese medical setting. Drugs Aging 2008;25:49-59

12. Veena D.R, Padma L, Sapna Patil. Drug prescribing pattern in elderly patients in a teaching hospital. IOSR Journal of Dental and Medical Sciences (JDMS). Volume 1, Issue 5 (Sep-Oct. 2012), PP 39-42

13. Harugeri A, Joseph J, Parthasarathi G, Ramesh M, Guido S. Potentially inappropriate medication use in elderly patients: A study of prevalence and predictors in two teaching hospitals. J Postgrad Med 2010;56:186-91.

14. Mandavi, Pramil Tiwari, Vinay Kapur. Inappropriate drug prescribing identified among Indian elderly hospitalized patients, Int J Risk Saf Med 2007; 19: 111-116

15. Klarin I,Wimo A, Fastbom J. The association of inappropriate drug use with hospitalisation and mortality: a population based study of the very old. Drugs Aging 2005; 22: 69-82.

16. Jano E, Aparasu RR. Healthcare outcomes associated with Beers criteria: A systematic review. Ann Pharmacother. 2007;41:438-447.

17. Rothberg et al. Potentially Inappropriate Medication Use in Hospitalized Elders, J Hosp Med 20083 :2 91-102

18. Viktil et al. Polypharmacy as commonly defined is an indicator of limited value in the assessment of drug-related problems, Br J Clin Pharmacol 63 :2 187-195

19. E.R. Hajjar et al. Polypharamcy in elderly patients. Am J Geriatr Pharmacother. 2007 Dec; 5(4):34551 
20. Shalini, Joshi MC et al. Study of polypharmacy and associated problems among elderly patients http://www.akspublication.com/ijmu 2012 January;7(1):35-9. Accessed Feb 19, 2014.

21. Cooney D, Pascuzzi K. Polypharmacy in the elderly: focus on drug interactions and adherence in hypertension. Clin Geriatr Med 2009;25:221-33.

22. Espino DV, Bazaldua OV, Palmer RF, et al. Suboptimal medication use and mortality in an older adult communitybased cohort: Results from the Hispanic EPESE Study. J Gerontol A Biol Sci Med Sci. 2006;61:170-175.

23. Berdot et al, Inappropriate medication use and risk of falls - A prospective study in a large community-dwelling elderly cohort, BMC Geriatr. 2009; 9: 30.

24. Magaziner J, Cadigan DA, Fedder DO, Hebel JR. Medication use and functional decline among community dwelling older women. J Aging Health. 1989;1: 470- 484.

25. Marcum and Gellad. Medication Adherence to Multidrug Regimens. Clin Geriatr Med 28 (2012) 287-300

26. Sehgal, Bajwa SJS, Vishal et al. Polypharmacy and Potentially Inappropriate Medication Use as the Precipitating Factor in Readmissions to the Hospital. J Family Med Prim Care. 2013 Apr-Jun; 2(2): 194-199.

27. Stacy et al, Potentially Inappropriate Prescribing of Benzodiazepines for Older Adults and Risk of Falls During a Hospital Stay: A Descriptive Study .Can J Hosp Pharm. 2009 Jul-Aug; 62(4): 276283.

28. Tsai R, Potentially Inappropriate Medication Use in Mild Cognitive Impairment (MCI) Patients: Results from the Kerala Einstein Study. J Am Geriatr Soc. 2012 July; 60(7): 1369-1370.

29. Barry et al, START (screening tool to alert doctors to the right treatment) — an evidence-based screening tool to detect prescribing omissions in elderly patients Age and Ageing 2007; 36: 632638.

30. Hamilton et al, Inappropriate prescribing and adverse drug events in older people. BMC Geriatrics 2009, 9:5

31. Naugler CT, Brymer C, Stolee P, Arcese ZA (2000) Development and validation of an improved prescribing for the elderly tool. The Canadian Journal of Clinical Pharmacology, 7, 103-107

32. M. Crotty, J Halbert, D Rowett et al. An outreach geriatric medication advisory service in residential aged care: a randomised controlled trial of case conferencing. Age Ageing 2004; 33: 612-617. 


\section{GRAPHICS}

Table 1: Clinical and demographic characteristics of study subjects $(n=150)$

Characteristics $^{1}$

Ag category

$$
\begin{gathered}
\text { 60-74 } \\
75-84 \\
>84
\end{gathered}
$$

Sex

$$
\text { Male }
$$

Female

Polypharmacy Used

$$
\begin{aligned}
& \text { Less than } 5 \\
& \text { More than } 5
\end{aligned}
$$

Number of PIMS

$$
\begin{aligned}
& 0 \\
& 1
\end{aligned}
$$$$
2
$$$$
3
$$

PIM

$$
\begin{aligned}
& \text { No } \\
& \text { Yes }
\end{aligned}
$$

Presence of co-morbidities

Presence of Diabetes

Presence of hypertension

Presence of chronic lung disease

Presence of IHD

Presence of BPH

Presence of stroke
Frequencies

$120(80.0)$

22 (14.7)

8 (5.3)

$56(37.3)$

$94(62.7)$

40 (26.7)

$110(73.3)$

$124(82.7)$

19 (12.7)

5 (3.3)

$2(1.3)$

$78(52.0)$

$80(53.3)$

$33(22.0)$

27 (18.0)

$13(8.7)$

$13(8.7)$ 
Presence of heart failure

Lower urinary tract symptoms

Presence of Parkinson's disease

Presence of seizures

Presence of peptic ulcer

Presence of delirium

History of falls or fractures

Present

Presence of Syncope

Presence of dementia

Presence of insomnia

Absent

Presence of urinary incontinence

Absent

Presence of constipation

Absent

Stress or mixed urinary incontinence

Absent

Duration of hospital stay*

$8.1 \pm 7.5$

$6(4,9)$

Total number of problems*

$4.3 \pm 2.2$

$4(3,6)$

$6.7 \pm 3.1$

Number of medicines Used*

$7(4,9)$

${ }^{1}$ Reported as number within parenthesis percentages; *-mean \pm SD, median $\left(25^{\text {th }} \& 75^{\text {th }}\right.$ percentile $)$ 
Table 2: Factors associated with poly -pharmacy used:

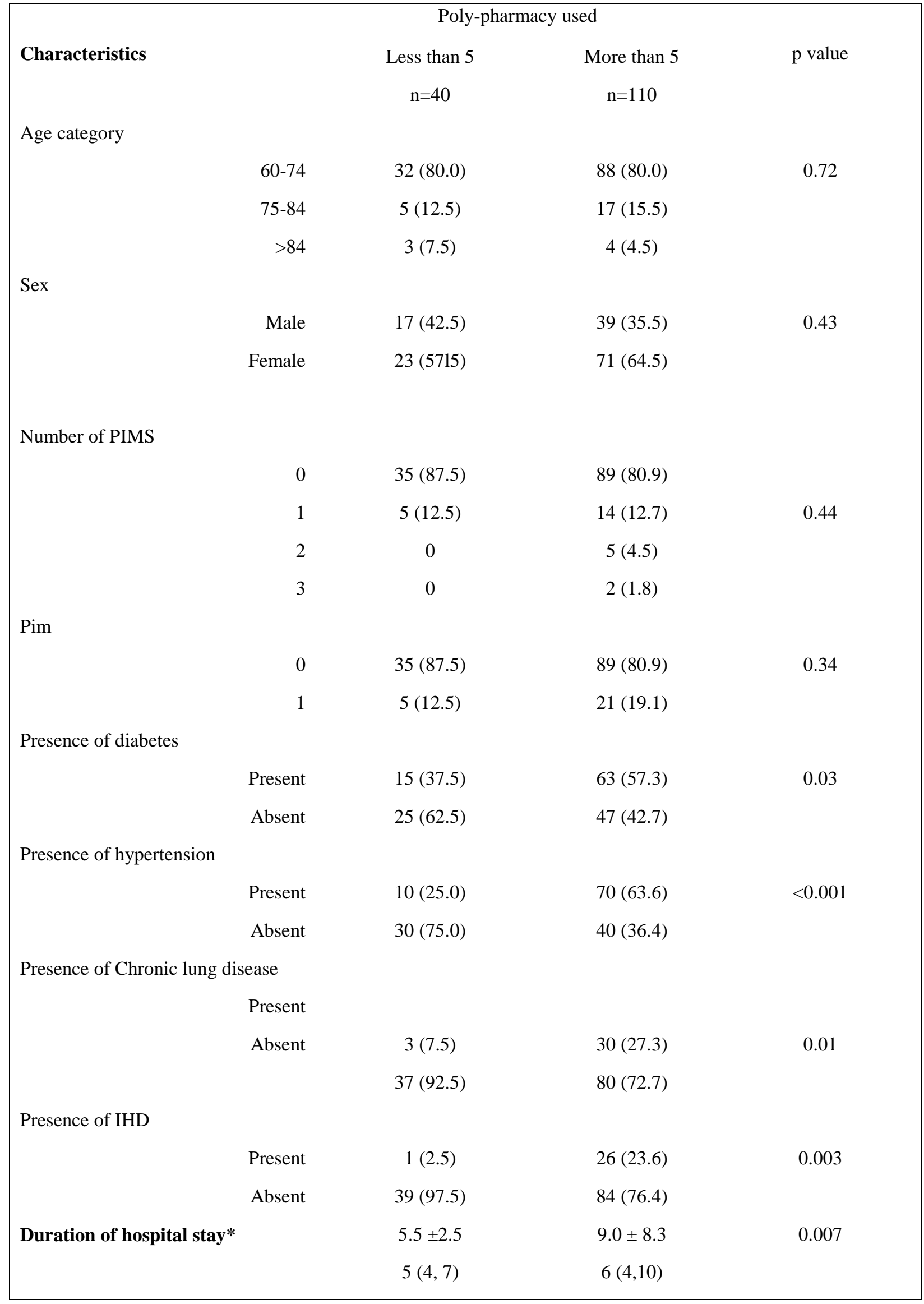




\begin{tabular}{|lccc|}
\hline Total number of problems* & $2.5 \pm 1.3$ & $4.9 \pm 2.1$ & $<0.001$ \\
& $3(1,3)$ & $5(3,6)$ & \\
Number of medicines used* & $3.1 \pm 0.92$ & $8.1 \pm 2.4$ & $<0.001$ \\
& $3(3,4)$ & $7(6,9)$ & \\
\hline
\end{tabular}

Reported as number within parenthesis percentages; $*$-mean \pm SD, median $\left(25^{\text {th }} \& 75^{\text {th }}\right.$ percentile $) ; p$ values using chi-square

Table 3: Factors Associated With PIM:

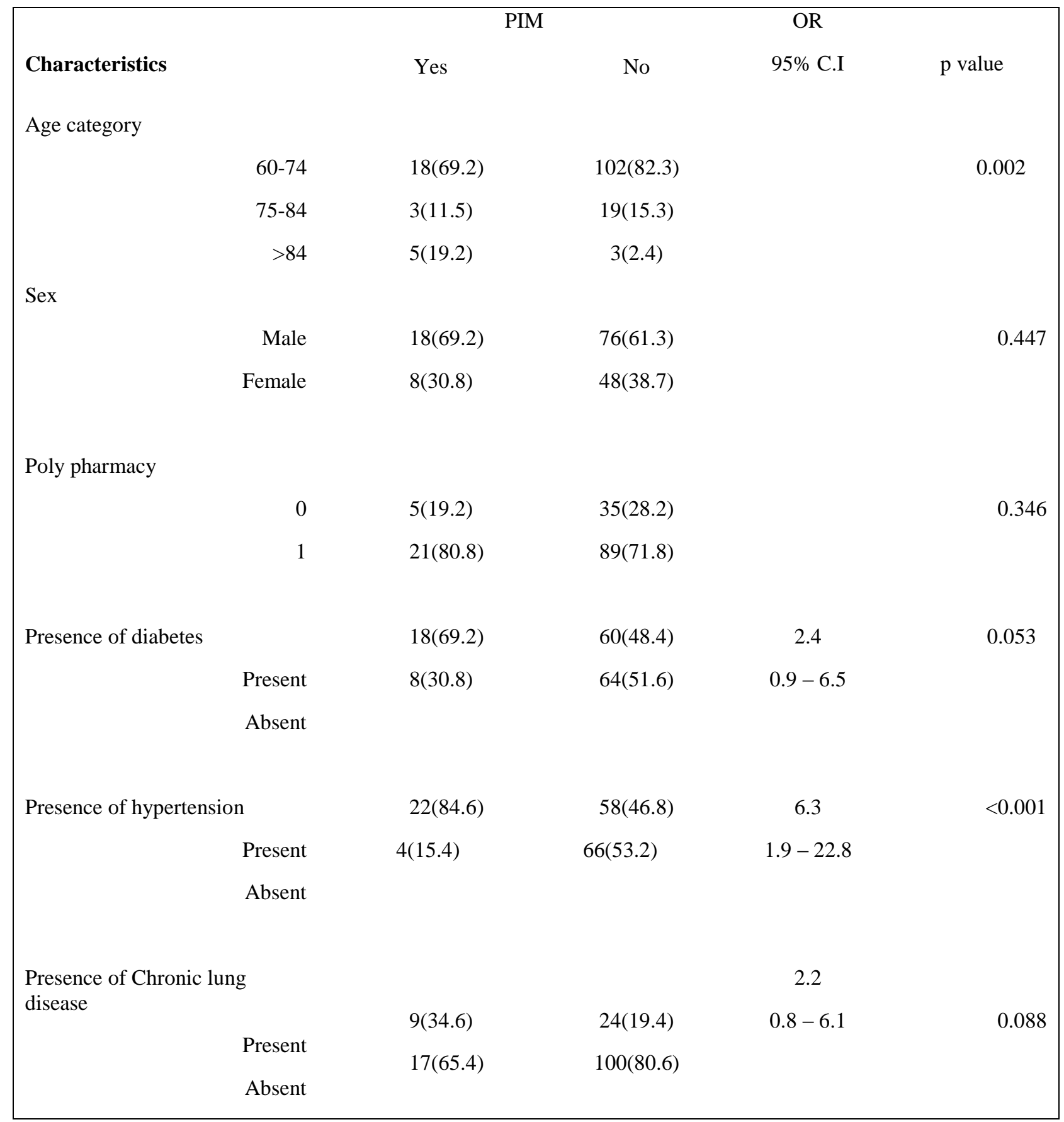




\begin{tabular}{|c|c|c|c|c|}
\hline Presence of IHD & $10(38.5)$ & 17(13.7) & 3.9 & 0.009 \\
\hline Present & $16(61.5)$ & $107(86.3)$ & $1.4-11.1$ & \\
\hline Absent & & & & \\
\hline \multirow[t]{2}{*}{ Duration of hospital stay* } & $8.7 \pm 6.54$ & $7.9 \pm 7.65$ & & 0.449 \\
\hline & $6.5(4,11)$ & $6(4,8)$ & & \\
\hline Total number of problems & $5.77 \pm 2.50$ & $3.97 \pm 2.05$ & & $<0.001$ \\
\hline \multirow[t]{2}{*}{ Number of medicines used* } & $6.4 \pm 2.97$ & $8.1 \pm 3.36$ & & 0.011 \\
\hline & $6(4,8)$ & $9(5.75,10.25)$ & & \\
\hline
\end{tabular}

Reported as number within parenthesis percentages; *-mean \pm SD, median $\left(25^{\text {th }} \& 75^{\text {th }}\right.$ percentile $) ; p$ values using chi-square

\section{APPENDIX I}

\section{BEERS' CRITERIA FOR POTENTIALLY INAPPROPRIATE MEDICATION USE IN OLDER ADULTS}

TABLE 1: 2012 AGS Beers Criteria for Potentially Inappropriate Medication Use in Older Adults

Organ System/ Therapeutic

Category/Drug(s)
Recommendation, Rationale, Quality of Evidence (QE) \& Strength of

Recommendation (SR)

Anticholinergics (excludes TCAs)

First-generation antihistamines

(as single agent or as part of combination products)

- Brompheniramine

- Carbinoxamine

- Chlorpheniramine

- Clemastine

- Cyproheptadine

- Dexbrompheniramine

- Dexchlorpheniramine

- Diphenhydramine (oral)

- Doxylamine

- Hydroxyzine

- Promethazine

- Triprolidine

Antiparkinson agents

- Benztropine (oral)

- Trihexyphenidyl
Avoid.

Highly anticholinergic; clearance reduced with advanced age, and tolerance develops when used as hypnotic; increased risk of confusion, dry mouth, constipation, and other anticholinergic effects/toxicity.

Use of diphenhydramine in special situations such as acute treatment of severe allergic reaction may be appropriate.

$\mathrm{QE}=$ High (Hydroxyzine and Promethazine), Moderate (All others); SR $=$ Strong

Avoid.

Not recommended for prevention of extrapyramidal symptoms with antipsychotics; more effective agents available for treatment of Parkinson disease.

$\mathrm{QE}=$ Moderate $; \mathrm{SR}=$ Strong 
Antispasmodics

- Belladonna alkaloids

- Clidinium-chlordiazepoxide

- Dicyclomine

- Hyoscyamine

- Propantheline

- Scopolamine

Antithrombotics

Dipyridamole, oral short-acting* (does not apply to the extended-release combination with aspirin)

Ticlopidine*

Anti-infective

Nitrofurantoin

Cardiovascular

Alpha1 blockers

- Doxazosin

- Prazosin

- Terazosin

Alpha agonists

- Clonidine

- Guanabenz*

- Guanfacine*

- Methyldopa*

- $\quad$ Reserpine $(>0.1 \mathrm{mg} / \text { day })^{*}$

Antiarrhythmic drugs (Class Ia, Ic, III)

- Amiodarone

- Dofetilide

- Dronedarone

- Flecainide

- Ibutilide

- Procainamide

- Propafenone

- Quinidine

- Sotalol

- Disopyramide*

Dronedarone
Avoid except in short-term palliative care to decrease oral secretions.

Highly anticholinergic, uncertain effectiveness.

$\mathrm{QE}=$ Moderate $; \mathrm{SR}=$ Strong

Avoid.

May cause orthostatic hypotension; more effective alternatives available; IV form acceptable for use in cardiac stress testing.

$\mathrm{QE}=$ Moderate $; \mathrm{SR}=$ Strong

Avoid.

Safer, effective alternatives available.

$\mathrm{QE}=$ Moderate; $\mathrm{SR}=$ Strong

Avoid for long-term suppression; avoid in patients with $\mathrm{CrCl}$ $<60 \mathrm{~mL} / \mathrm{min}$.

Potential for pulmonary toxicity; safer alternatives available; lack of efficacy in patients with $\mathrm{CrCl}<60 \mathrm{~mL} / \mathrm{min}$ due to inadequate drug concentration in the urine.

$\mathrm{QE}=$ Moderate $; \mathrm{SR}=$ Strong

Avoid use as an antihypertensive.

High risk of orthostatic hypotension; not recommended as routine treatment for hypertension; alternative agents have superior risk/benefit profile.

$\mathrm{QE}=$ Moderate; $\mathrm{SR}=$ Strong

Avoid clonidine as a first-line antihypertensive. Avoid others as listed.

High risk of adverse CNS effects; may cause bradycardia and orthostatic hypotension; not recommended as routine treatment for hypertension.

$\mathrm{QE}=$ Low; $\mathrm{SR}=$ Strong

Avoid antiarrhythmic drugs as first-line treatment of atrial fibrillation.

Data suggest that rate control yields better balance of benefits and harms than rhythm control for most older adults.

Amiodarone is associated with multiple toxicities, including thyroid disease, pulmonary disorders, and QT interval prolongation.

$\mathrm{QE}=$ High; $\mathrm{SR}=$ Strong

Avoid.

Disopyramide is a potent negative inotrope and therefore may induce heart failure in older adults; strongly anticholinergic; other antiarrhythmic drugs preferred.

$\mathrm{QE}=$ Low; SR = Strong

Avoid in patients with permanent atrial fibrillation or heart failure.

Worse outcomes have been reported in patients taking dronedarone who have permanent atrial fibrillation or heart failure. In general, rate control is preferred over rhythm control for atrial fibrillation.

$\mathrm{QE}=$ Moderate; $\mathrm{SR}=$ Strong 
Digoxin $>0.125 \mathrm{mg} /$ day

Nifedipine, immediate release*

Spironolactone $>25 \mathrm{mg} /$ day

Central Nervous System

Tertiary TCAs, alone or in combination:

- Amitriptyline

- Chlordiazepoxide-

- Amitriptyline

- Clomipramine

- Doxepin >6 mg/day

- Imipramine

- Perphenazine-amitriptyline

- Trimipramine

Antipsychotics, first- (conventional) and second(atypical) generation (see online for full list)

Thioridazine

Mesoridazine

Barbiturates

- Amobarbital*

- Butabarbital*

- Butalbital

- Mephobarbital*

- Pentobarbital*

- Phenobarbital

- Secobarbital*

\section{Avoid.}

In heart failure, higher dosages associated with no additional benefit and may increase risk of toxicity; decreased renal clearance may increase risk of toxicity.

$\mathrm{QE}=$ Moderate $; \mathrm{SR}=$ Strong

Avoid.

Potential for hypotension; risk of precipitating myocardial ischemia.

$\mathrm{QE}=$ High; SR = Strong

Avoid in patients with heart failure or with a $\mathrm{CrCl}<30 \mathrm{~mL} / \mathrm{min}$. In heart failure, the risk of hyperkalemia is higher in older adults if taking $>25 \mathrm{mg} /$ day.

$\mathrm{QE}=$ Moderate; $\mathrm{SR}=$ Strong

Avoid.

Highly anticholinergic, sedating, and cause orthostatic hypotension; the safety profile of low-dose doxepin $(\leq 6$ $\mathrm{mg}$ /day) is comparable to that of placebo.

$\mathrm{QE}=$ High; SR = Strong

Avoid use for behavioral problems of dementia unless nonpharmacologic options have failed and patient is threat to self or others.

Increased risk of cerebrovascular accident (stroke) and mortality in persons with dementia.

$\mathrm{QE}=$ Moderate; $\mathrm{SR}=$ Strong

Avoid.

Highly anticholinergic and greater risk of QT-interval prolongation.

$\mathrm{QE}=$ Moderate $; \mathrm{SR}=$ Strong

Avoid.

High rate of physical dependence; tolerance to sleep benefits; greater risk of overdose at low dosages.

$\mathrm{QE}=$ High; $\mathrm{SR}=$ Strong 


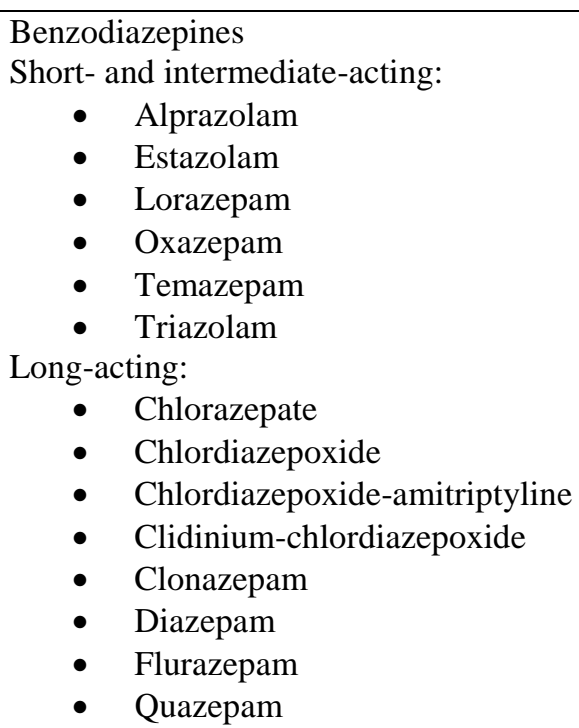

Chloral hydrate

Meprobamate

Nonbenzodiazepine

hypnotics

- Eszopiclone

- Zolpidem

- Zaleplon

Ergot mesylates*

Isoxsuprine*

Endocrine

Androgens

- Methyltestosterone*

- Testosterone

Desiccated thyroid

Estrogens with or without progestins
Avoid benzodiazepines (any type) for treatment of insomnia agitation, or delirium.

Older adults have increased sensitivity to benzodiazepines and decreased metabolism of long-acting agents. In general, all benzodiazepines increase risk of cognitive impairment, delirium, falls, fractures, and motor vehicle accidents in older adults. May be appropriate for seizure disorders, rapid eye movement sleep disorders, benzodiazepine withdrawal, ethanol withdrawal, severe generalized anxiety disorder, periprocedural anesthesia, end-of-life care.

$\mathrm{QE}=$ High; SR = Strong

Avoid.

Tolerance occurs within 10 days and risk outweighs the benefits in light of overdose with doses only 3 times the recommended dose.

$\mathrm{QE}=$ Low; SR = Strong

Avoid.

High rate of physical dependence; very sedating.

$\mathrm{QE}=$ Moderate; $\mathrm{SR}=$ Strong

Avoid chronic use ( $>90$ days)

Benzodiazepine-receptor agonists that have adverse events similar to those of benzodiazepines in older adults (e.g., delirium, falls, fractures); minimal improvement in sleep latency and duration.

$\mathrm{QE}=$ Moderate $; \mathrm{SR}=$ Strong

Avoid.

Lack of efficacy.

$\mathrm{QE}=$ High; SR = Strong

Avoid unless indicated for moderate to severe hypogonadism.

Potential for cardiac problems and contraindicated in men with prostate cancer.

$\mathrm{QE}=$ Moderate $; \mathrm{SR}=$ Weak

Avoid.

Concerns about cardiac effects; safer alternatives available.

$\mathrm{QE}=$ Low; SR = Strong

Avoid oral and topical patch. Topical vaginal cream: Acceptable to use low-dose intravaginal estrogen for the management of dyspareunia, lower urinary tract infections, and other vaginal symptoms.

Evidence of carcinogenic potential (breast and endometrium); lack of cardioprotective effect and cognitive protection in older women.

Evidence that vaginal estrogens for treatment of vaginal dryness is safe and effective in women with breast cancer, especially at dosages of estradiol $<25 \mathrm{mcg}$ twice weekly.

$\mathrm{QE}=$ High (Oral and Patch), Moderate (Topical); SR = Strong (Oral and Patch), Weak (Topical) 
Growth hormone

Insulin, sliding scale

Megestrol

Sulfonylureas, long-duration

- Chlorpropamide

- Glyburide

Gastrointestinal

Metoclopramide

Mineral oil, given orally

Trimethobenzamide

Pain Medications

Meperidine

Non-COX-selective NSAIDs, oral

- Aspirin >325 mg/day

- Diclofenac

- Diflunisal

- Etodolac

- Fenoprofen

- Ibuprofen

- Ketoprofen

- Meclofenamate

- Mefenamic acid

- Meloxicam

- Nabumetone

- Naproxen

- Oxaprozin

- Piroxicam

- Sulindac

- Tolmetin
Avoid, except as hormone replacement following pituitary gland removal.

Effect on body composition is small and associated with edema, arthralgia, carpal tunnel syndrome, gynecomastia, impaired fasting glucose.

$\mathrm{QE}=$ High; SR = Strong

Avoid.

Higher risk of hypoglycemia without improvement in hyperglycemia management regardless of care setting. $\mathrm{QE}=$ Moderate; $\mathrm{SR}=$ Strong

Avoid.

Minimal effect on weight; increases risk of thrombotic events and possibly death in older adults.

$\mathrm{QE}=$ Moderate; $\mathrm{SR}=$ Strong

Avoid.

Chlorpropamide: prolonged half-life in older adults; can cause prolonged hypoglycemia; causes SIADH

Glyburide: higher risk of severe prolonged hypoglycemia in older adults.

$\mathrm{QE}=$ High; SR = Strong

Avoid, unless for gastroparesis.

Can cause extrapyramidal effects including tardive dyskinesia; risk may be further increased in frail older adults.

$\mathrm{QE}=$ Moderate; $\mathrm{SR}=$ Strong

Avoid.

Potential for aspiration and adverse effects; safer alternatives available.

$\mathrm{QE}=$ Moderate; $\mathrm{SR}=$ Strong

Avoid.

One of the least effective antiemetic drugs; can cause

extrapyramidal adverse effects.

$\mathrm{QE}=$ Moderate; $\mathrm{SR}=$ Stron

Avoid.

Not an effective oral analgesic in dosages commonly used; may cause neurotoxicity; safer alternatives available.

$\mathrm{QE}=$ High; $\mathrm{SR}=$ Strong

Avoid chronic use unless other alternatives are not effective and patient can take gastroprotective agent (proton-pump inhibitor or misoprostol).

Increases risk of GI bleeding/peptic ulcer disease in high-risk groups, including those $\geq 75$ years old or taking oral or parenteral corticosteroids, anticoagulants, or antiplatelet agents. Use of proton pump inhibitor or misoprostol reduces but does not eliminate risk.

$\mathrm{QE}=$ Moderate $; \mathrm{SR}=$ Strong 
- Indomethacin

- Ketorolac, includes parenteral

Pentazocine*

Skeletal muscle relaxants

- Carisoprodol

- Chlorzoxazone

- Cyclobenzaprine

- Metaxalone

- Methocarbamol

- Orphenadrine

\section{Avoid.}

Increases risk of GI bleeding/peptic ulcer disease in high-risk groups (See Non-COX selective NSAIDs)

Of all the NSAIDs, indomethacin has most adverse effects.

$\mathrm{QE}=$ Moderate (Indomethacin), High (Ketorolac); SR = Strong Avoid.

Opioid analgesic that causes CNS adverse effects, including confusion and hallucinations, more commonly than other narcotic drugs; is also a mixed agonist and antagonist; safer alternatives available.

$\mathrm{QE}=$ Low; SR = Strong

Avoid.

Most muscle relaxants poorly tolerated by older adults, because of anticholinergic adverse effects, sedation, increased risk of fractures; effectiveness at dosages tolerated by older adults is questionable.

$\mathrm{QE}=$ Moderate; $\mathrm{SR}=$ Strong

*Infrequently used drugs. Table 1 Abbreviations: ACEI, angiotensin converting-enzyme inhibitors; ARB, angiotensin receptor blockers; CNS, central nervous system; COX, cyclooxygenase; $\mathrm{CrCl}$, creatinine clearance; GI, gastrointestinal; NSAIDs, nonsteroidal anti-inflammatory drugs; SIADH, syndrome of inappropriate antidiuretic hormone secretion; SR, Strength of Recommendation; TCAs, tricyclic antidepressants; QE, Quality of Evidence

TABLE 2: 2012 AGS Beers Criteria for Potentially Inappropriate Medication Use in Older Adults Due to Drug-Disease or Drug-Syndrome Interactions That May Exacerbate the Disease or Syndrome

\begin{tabular}{lcc} 
Disease or Syndrome & Drug(s) & $\begin{array}{c}\text { Recommendation, Rationale, Quality of } \\
\text { Evidence (QE) \& Strength of } \\
\text { Recommendation (SR) }\end{array}$ \\
\hline
\end{tabular}

Cardiovascular

Heart failure

Syncope

Central Nervous System

Chronic seizures or epilepsy
NSAIDs and COX-2 inhibitors Nondihydropyridine CCBs (avoid only for systolic heart failure)

- Diltiazem

- Verapamil

Pioglitazone, rosiglitazone

Cilostazol

Dronedarone

Acetylcholinesterase inhibitors

(AChEIs)

Peripheral alpha blockers

- Doxazosin

- Prazosin

- Terazosin

Tertiary TCAs

Chlorpromazine, thioridazine, and olanzapine
Bupropion
Chlorpromazine
Clozapine
Maprotiline
Olanzapine
Thioridazine
Thiothixene
Tramadol

Avoid.

Potential to promote fluid retention and/or exacerbate heart failure.

$\mathrm{QE}=$ Moderate (NSAIDs, CCBs,

Dronedarone), High (Thiazolidinediones (glitazones)), Low (Cilostazol); SR = Strong

Avoid.

Increases risk of orthostatic hypotension or bradycardia.

$\mathrm{QE}=$ High (Alpha blockers), Moderate

(AChEIs, TCAs and antipsychotics); SR = Strong (AChEIs and TCAs), Weak (Alpha blockers and antipsychotics)

Avoid.

Lowers seizure threshold; may be acceptable in patients with well-controlled seizures in whom alternative agents have not been effective.

$\mathrm{QE}=$ Moderate $; \mathrm{SR}=$ Strong 


\begin{tabular}{|c|c|}
\hline Delirium & $\begin{array}{l}\text { All TCAs } \\
\text { Anticholinergics Benzodiazepines } \\
\text { Chlorpromazine } \\
\text { Corticosteroids } \\
\text { H2-receptor antagonist } \\
\text { Meperidine } \\
\text { Sedative hypnotics } \\
\text { Thioridazine }\end{array}$ \\
\hline Dementia \& cognitive impairment & $\begin{array}{l}\text { Anticholinergics Benzodiazepines } \\
\mathrm{H} 2 \text {-receptor antagonists } \\
\text { Zolpidem } \\
\text { Antipsychotics, chronic and as- } \\
\text { needed use }\end{array}$ \\
\hline History of falls or fractures & $\begin{array}{l}\text { Anticonvulsants } \\
\text { Antipsychotics } \\
\text { Benzodiazepines } \\
\text { Nonbenzodiazepine hypnotics } \\
\text { - Eszopiclone } \\
\text { - Zaleplon } \\
\text { - Zolpidem } \\
\text { TCAs/SSRIs }\end{array}$ \\
\hline Insomnia & $\begin{array}{l}\text { Oral decongestants } \\
\text { - Pseudoephedrine } \\
\text { - Phenylephrine Stimulants } \\
\text { - Amphetamine } \\
\text { - Methylphenidate } \\
\text { - Pemoline Theobromines } \\
\text { - Theophylline } \\
\text { - Caffeine }\end{array}$ \\
\hline Parkinson's disease & $\begin{array}{l}\text { All antipsychotics (see online } \\
\text { publication for full list, except for } \\
\text { quetiapine and clozapine) } \\
\text { Antiemetics } \\
\text { - Metoclopramide } \\
\text { - Prochlorperazine } \\
\text { - Promethazine }\end{array}$ \\
\hline
\end{tabular}

Avoid.

Avoid in older adults with or at high risk of delirium because of inducing or worsening delirium in older adults; if discontinuing drugs used chronically, taper to avoid withdrawal symptoms.

$\mathrm{QE}=$ Moderate $; \mathrm{SR}=$ Strong

Avoid.

Avoid due to adverse CNS effects.

Avoid unless safer alternatives are not available; avoid anticonvulsants except for seizure.

Ability to produce ataxia, impaired psychomotor function, syncope, and additional falls; shorter-acting benzodiazepines are not safer than longacting ones.

$\mathrm{QE}=$ High; SR = Strong

Avoid.

CNS stimulant effects.

$\mathrm{QE}=$ Moderate $; \mathrm{SR}=$ Strong

Avoid.

Dopamine receptor antagonists with potential to worsen parkinsonian symptoms.

Quetiapine and clozapine appear to be less likely to precipitate worsening of Parkinson disease.

$\mathrm{QE}=$ Moderate $; \mathrm{SR}=$ Strong

Gastrointestinal 
Chronic constipation

History of gastric or duodenal ulcers

Kidney/Urinary Tract

Chronic kidney disease stages IV and $\mathrm{V}$

Urinary incontinence (all types) in women

Lower urinary tract symptoms, benign prostatic hyperplasia

Stress or mixed urinary incontinence
Oral antimuscarinics for urinary incontinence

- Darifenacin

- Oxybutynin (oral)

- Solifenacin

- Tolterodine

- Trospium

- Nondihydropyridine CCB

- Diltiazem

- Verapamil

First-generation antihistamines as single agent or part of combination products

- Brompheniramine

- Chlorpheniramine

- Cyproheptadine

- Dexchlorpheniramine

- Diphenhydramine

- Doxylamine

- Hydroxyzine

- Promethazine

Anticholinergics/antispasmodics

- Antipsychotics

- Belladonna alkaloids

- Clidinium-chlordiazepoxide

- Dicyclomine

- Hyoscyamine

- Propantheline

- Scopolamine

- Tertiary TCAs

Aspirin (>325 mg/day)

Non-COX-2 selective NSAIDs

NSAIDs

Triamterene (alone or in combination)

Estrogen oral and transdermal (excludes intravaginal estrogen)

Inhaled anticholinergic agents Strongly anticholinergic drugs, except antimuscarinics for urinary incontinence (see Table 9 for complete list).

Alpha-blockers

- Doxazosin

- Prazosin

- Terazosin
Avoid unless no other alternatives.

Can worsen constipation; agents for urinary incontinence: antimuscarinics overall differ in incidence of constipation; response variable; consider alternative agent if constipation develops.

$\mathrm{QE}=$ High (For Urinary Incontinence), Moderate/Low (All Others); SR = Strong
Avoid unless other alternatives are not effective and patient can take gastroprotective agent (proton-pump inhibitor or misoprostol). May exacerbate existing ulcers or cause new/additional ulcers.

$\mathrm{QE}=$ Moderate; $\mathrm{SR}=$ Strong

Avoid.

May increase risk of kidney injury.

May increase risk of acute kidney injury.

$\mathrm{QE}=$ Moderate (NSAIDs), Low

(Triamterene); SR = Strong (NSAIDs),

Weak (Triamterene)

Avoid in women.

Aggravation of incontinence.

$\mathrm{QE}=$ High; SR = Strong

Avoid in men.

May decrease urinary flow and cause urinary retention.

$\mathrm{QE}=$ Moderate $; \mathrm{SR}=$ Strong (Inhaled agents), Weak (All others)

Avoid in women.

Aggravation of incontinence.

$\mathrm{QE}=$ Moderate $; \mathrm{SR}=$ Strong 
Table 2 Abbreviations: CCBs, calcium channel blockers; AChEIs, acetylcholinesterase inhibitors; CNS, central nervous system; COX, cyclooxygenase; NSAIDs, nonsteroidal anti-inflammatory drugs; SR, Strength of Recommendation; SSRIs, selective serotonin reuptake inhibitors; TCAs, tricyclic antidepressants; QE, Quality of Evidence

TABLE 3: 2012 AGS Beers Criteria for Potentially Inappropriate Medications to Be Used with Caution in Older Adults

Drug(s) Recommendation, Rationale, Quality of Evidence (QE) \&

Aspirin for primary prevention of cardiac events

Dabigatran

Prasugrel

Antipsychotics

Carbamazepine

Carboplatin

Cisplatin

Mirtazapine

SNRIs

SSRIs

TCAs

Vincristine

Vasodilators Strength of Recommendation (SR)

Use with caution in adults $\geq 80$ years old.

Lack of evidence of benefit versus risk in individuals $\geq 80$ years old.

$\mathrm{QE}=$ Low $; \mathrm{SR}=$ Weak

Use with caution in adults $\geq 75$ years old or if $\mathrm{CrCl}<30$ $\mathrm{mL} / \mathrm{min}$.

Increased risk of bleeding compared with warfarin in adults $\geq 75$ years old; lack of evidence for efficacy and safety in patients with $\mathrm{CrCl}<30 \mathrm{~mL} / \mathrm{min}$

$\mathrm{QE}=$ Moderate; $\mathrm{SR}=$ Weak

Use with caution in adults $\geq 75$ years old.

Greater risk of bleeding in older adults; risk may be offset by benefit in highest-risk older patients (eg, those with prior myocardial infarction or diabetes).

$\mathrm{QE}=$ Moderate; $\mathrm{SR}=$ Weak

Use with caution.

May exacerbate or cause SIADH or hyponatremia; need to monitor sodium level closely when starting or changing dosages in older adults due to increased risk.

$\mathrm{QE}=$ Moderate $; \mathrm{SR}=$ Strong

Use with caution.

May exacerbate episodes of syncope in individuals with history of syncope.

$\mathrm{QE}=$ Moderate; $\mathrm{SR}=\mathrm{Weak}$

Table 3 Abbreviations: $\mathrm{CrCl}$, creatinine clearance; SIADH, syndrome of inappropriate antidiuretic hormone 